\title{
THE STUDY ON THE ACTIVITY OF THE HYPOTHALAMUS AND THE LIMBIC SYSTEM UNDER EXTREME COLD
}

\author{
Masazumi Kawakami, Hideo Negoro, Masahiro Yanase \\ AND Motohiko MOHRI \\ 2nd Department of Physiology \\ Yokohama City University School of Medicine, Yokohama
}

The study of the central control mechanism of body temperature has yielded remarkable results since the experiment by Aronsohn and Sachs using the method of heat puncture in 1884. The existence of receptors for body temperature in the anterior hypothalamus was found by BARBOURS ${ }^{1)}$, RANSON and MAGOUN ${ }^{2)}$ and HARDY $^{3)}$ according to the method of local heating and cooling of the brain. Recently NAKAyAma et al. ${ }^{4)}$ and MuraKami et al. ${ }^{5)}$ have reported that local heating at the anterior hypothalamus has resulted in an increase in the unit activity of cells in the anterior hypothalamus. ANDERSON $\left.{ }^{6}, 7\right)$ also has reported on the appearance of panting or disappearance of shivering, caused by stimulation of the anterior hypothalamus of the unanesthetized goat. On the other hand, many studies such as those by HEMINGWAY ${ }^{8)}$ have been carried out on the mechanism of shivering, an involuntary movement which is an effective source of heat supply. The mechanism of temperature control by the anterior hypothalamic area has been gradually clarified. OGATA and KATAYAMA ${ }^{9)}$ and KoIKEGAMi ${ }^{10)}$ reported that the stimulation of amygdala resulted in rise of body temperature. However, the role of the limbic system in controlling body temperature by means of the hypothalamus is not as yet well known. This study aims at elucidation of the role and activity of the limbic area in addition to hypothalamic activity exposure to extreme cold. It is now well understood that activity of the limbic system and hypothalamus has a great influence upon endocrine function as well as autonomic function. The previous studies on the maintenance and control of body temperature have been concerned mainly with its neurological aspects. In this experiment, the authors tried to make clear the control mechanism of body temperature from the viewpoint of neurohumoral interaction; that is, the interrelationship between brain activity and internal secretion.

Received for publication October 16, 1968

川上正澄, 根来英雄, 柳瀬昌弘, 毛利元彦 


\section{MATERIALS AND METHODS}

One hundred and seventy-three mature New Zealand white rabbits and $28 \mathrm{im}$ mature were employed. For "chronic" recording, permanent bipolar concentric electrodes made of stainless steel tube and cores, insulated by Formvar, except at the tip, were implanted in the brain stereotaxically. Electrodes were fixed in position by acrylic resin attached to the calvarium and to screws embedded in the skull. Silver ball electrodes resting on the dura were used for cortical records.

Twenty to 25 days later, when the rabbits recovered from the post-operative invasion, they were placed in a sound-proof room with constant illumination. EEG recording was carried out from several parts of the brain at an arousal stage using an electroencephalograph (Sanei, EG-900). During EEG recording the rabbit was free to move around on an observation table or in a cold box, to eat, drink and sleep; thus all the acute stress factors except cold were excluded. The EEG was analyzed with a band-pass type analyzer (Sanei, EA-201) into 5 frequency bands, such as 2-4 cps, $4-8 \mathrm{cps}, 8-13 \mathrm{cps}, 13-20 \mathrm{cps}$ and $20-30 \mathrm{cps}$; each analysis period being 70 seconds.

Cold exposure was applied by placing the animal in a cold box in which it was possible to control the temperature.

Stimulation consisted of monophasic square wave pulses delivered unilaterally with a Sanei stimulator (ES-103) and isolation unit for one hour, $60 \mathrm{sec}$. on and $60 \mathrm{sec}$. off, at $0.5 \mathrm{msec}$. duration, $100 \mathrm{cps}$. EEG and EMG were recorded during stimulation and inter-stimulation period to detect whether or not an EEG seizure pattern and EMG grouping discharge were induced by the stimulation.

The position of the electrode implants in the brain were examined histologically after the experiment. EEG recording and stimulation of each part of the brain were performed using 6 to 12 rabbits respectively. For the administration of adrenocortical hormone and thyroid hormone, hydrocortisone acetate (Merck \& Co., Inc.-U.S. A.) and 1-thyroxine $\mathrm{Na}$ (Teikoku zoki \& Co.) were used. Adrenalectomized rabbits were used for the experiment 4 days after the operation so that the accessory adrenocortical deposits would not develop, while thyroidectomized rabbits were used more than 3 weeks after the operation, when previously secreted thyroid hormone must have been completely consumed.

\section{RESULTS}

The rabbits with chronically implanted electrodes in various brain areas were exposed to extreme cold $\left(-20^{\circ} \mathrm{C}\right)$ for 12 hours and the change in EEG activity of the limbic system and other related structures was studied. It was found that the change in rectal temperature of the rabbit under cold exposure shows some seasonal variation. In summer the rectal temperature gradually declined during cold exposure. After 6-10 hours of exposure the temperature declined by $0.7 \pm 0.3^{\circ} \mathrm{C}$. In the cold box the animal sat quietly without moving except when eating or drinking. On the other hand, in winter the rectal temperature rose by $0.5 \pm 0.4^{\circ} \mathrm{C}$ during cold exposure and the animal was rather restless, scratching the wall of the cold box and sniffing around, while squatting or standing.

1. Electrical activity of the limbic system under exposure to extreme cold. 


\section{Hippocampus}

The basic EEG of the hippocampus in arousal state was a sinusoidal wave overlapped by a high frequency wave of low amplitude so that the dominant frequency component under normal conditions consisted of $4-8 \mathrm{cps}$. In mid-summer this component was most remarkably increased during cold exposure (FIG. 1). More precisely, the $4-8 \mathrm{cps}$ component began to increase in amplitude 30 minutes after starting cold exposure and the maximum was attained in the next 30 minutes. Then this component began to decline, but

\section{HIPPOCAMPUS}

SUMMER
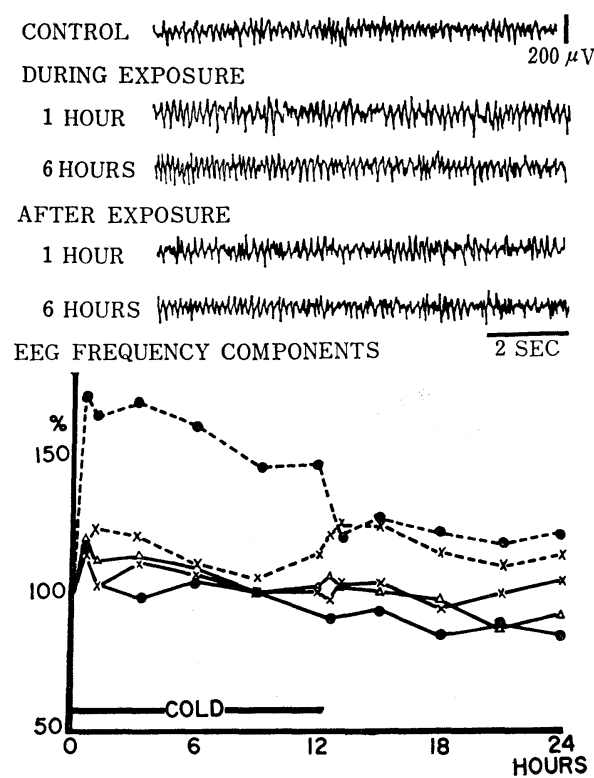

WINTER

FIG. 1. Typical example of the change in EEG activity (the upper party) of the hippocampus and its frequency components (lower part) during and after cold exposure $\left(-20^{\circ} \mathrm{C}\right)$.

Ordinate: Increasing rate of integrated band value for 10 seconds in percentage of the control period before the cold exposure.

Abscissa: Time in hours after cold exposure.

Note that in summer, theta wave of $4-8 \mathrm{cps}$ was increased in amplitude and was synchronized during cold exposure, while in winter hippocampal EEG pattern was not activated cold exposure.

the control level was not restored by the 12th hour of cold exposure. When the animal was brought back to the normal environment of $22^{\circ} \mathrm{C}$, the control level was restored within one hour. The $2-4 \mathrm{cps}$ component was found to decrease and the 8-30 cps component increased, though they were non-significant statistically. The hippocampal EEG activation was also observed in 
the spring and autumn during cold exposure, but the change was milder. On the contrary, in the mid-winter, cold exposure induced little change in hippocampal EEG activity.

\section{Central grey}

The basic wave of the central grey was characterized by a rhythmical sinusoidal wave of $4-8 \mathrm{cps}$. It was enhanced under exposure to extreme cold. During the first half of the cold exposure, the synchronization and the amplitude of the theta wave were enhanced most remarkably. In 7 out of 9 examples the $4-8 \mathrm{cps}$ component increased by $16-30 \%$ (av. $25 \%$ ) in integrated value. (10 seconds) after 3 to 9 hours of cold exposure. Then it gradually decreased, and after 12 hours of cold exposure returned to the control level. The $8-13$ cps component hardly changed, although it increased a small amount. On the other hand, $2-4 \mathrm{cps}$ and $13-20 \mathrm{cps}$ frequency components showed a tendency to decrease. Two of the 9 examples showed a gradual increase of all frequency components after they were exposed to extreme cold, and reached their peak after 12 hours of cold exposure. The 4-8cps theta wave showed a remarkable increase of $25-30 \%$ in integrated value at the 12 hours. When removed from the cold exposure all of the 9 examples returned within an hour to the control state. In mid-summer the basic $4-8 \mathrm{cps}$ wave of the central grey under cold exposure increased in rhythmicity and amplitude remarkably, while in winter 4 out of 8 examples hardly changed and the amplitude of the wave was decreased in 2 of 6 examples. Therefore, the results showed that the activity level of the central grey was enhanced under cold exposure and that this was especially remarkable in summer, while it was less obvious or declined in winter.

\section{Septum}

Opposite responses to cold exposure were observed between EEGs recorded from the medial part and the lateral part of the septum.

As for the activity of the medial septum, the amplitude of the wave was decreased both in summer and winter under cold exposure, but in midsummer the change was the greatest.

The EEG of the medial septum consists of a $4-8 \mathrm{cps}$ theta wave superimposing on the $20-30 \mathrm{cps}$ component. When the rabbits were exposed to extreme cold, all frequency components decreased. These changes appeared after 30 minutes of cold exposure, and they retained the level for 12 hours of cold exposure. When the animal was brought back to the normal environment, the 4-8cps component was increased above the control level for about 30 minutes and then it gradually recovered; in an hour it returned to the control level.

As for the EEG of the lateral septum, fast wave is the dominant component and is mixed with irregular slow waves in arousal state. When the rabbit was exposed to cold, regular rhythmic theta waves of $4-8 \mathrm{cps}$ domina- 
ted over other frequency components. The integrated value of $4-8 \mathrm{cps}$ and $20-30$ cps frequency components increased about $20-30 \%$ and $10-28 \%$ respectively for 6 hours of cold exposure in summer. And then every frequency component began to decrease in integrated value. After 10 hours of cold exposure, all the frequency components were reduced by between 8 and 10\% of the controls. Thus the activity of the medial septum was suppressed by cold exposure, while activity of the lateral septum was enhanced during the early phase of cold exposure. However, the activity of the lateral septum began to be suppressed after prolonged exposure (between 6-12 hours) to cold. In winter,enhancement of activity of the lateral septum during the early phase of cold exposure was less relevant than in other seasons, and was seen only at the beginning of cold exposure.

\section{Amygdala}

As for the EEG recorded from the medial amygdala, slow wave components decreased remarkably under cold exposure, but fast components of $20-30 \mathrm{cps}$ (the basic component) gradually increased. This increase lasted for more than 3 hours, even after the animal was released from cold exposure.

MED. AMYGDALA

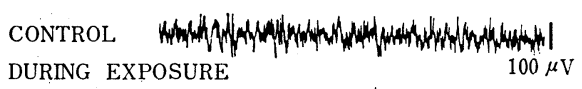

DURING EXPOSURE

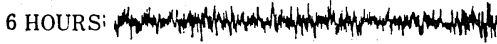

12 HOURS AFTER EXPOSURE

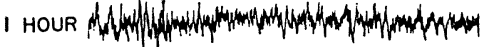

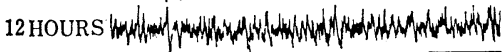

EEG FREQUENCY COMPONENTS

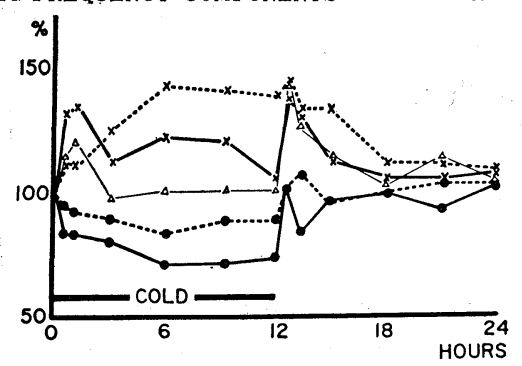

\section{LAT. AMYGDALA}

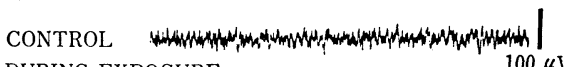

DURING EXPOSURE

$100 \mu \mathrm{V}$

12 HOURS

AFTER EXPOSURE

1 HOUR

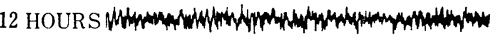
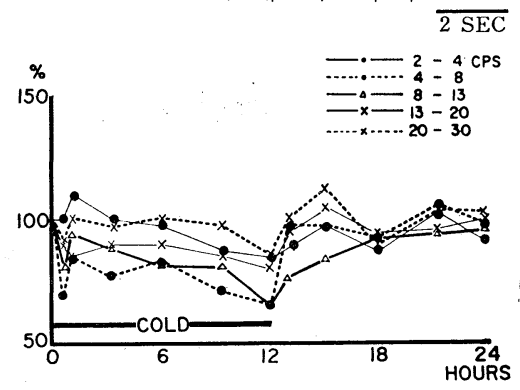

FIG. 2. The change in EEG and its frequency components of the medial amygdala and lateral amygdala during and after cold exposure.

Ordinate: Increasing rate of integrated band value for 10 seconds in percentage of the control period before the cold exposure.

Abscissa: Time in hours after cold exposure.

Note the gradual increase of fast wave component during cold exposure in the medial amygdala and decrease of almost all EEG frequency components during exposure in the lateral amygdala. 
Other frequency band components showed a tendency to decrease slightly during cold exposure. Amygdalar activity was unique in that such a heightened activity continued even when the animal was no longer exposed to cold. EEG recorded from the lateral amygdala showed a decrease of amplitude by cold exposure and all frequency components were decreased in amplitude. Thus, from the EEG pattern, medial and lateral amygdala show opposite changes under cold exposure (FIG. 2).

2. Electrical activity of the hypothalamus under exposure to extreme cold.

Posterior hypothalamus

The main frequency component of the integrated values of the posterior hypothalamus, considered to be a center of heat production by RANSON and others, consisted of $4-8 \mathrm{cps}$. This component was increased 12 to $30 \%$ after an hour of cold exposure and the EEG pattern showed synchronized high

\section{POST. HYPOTHALAMUS}
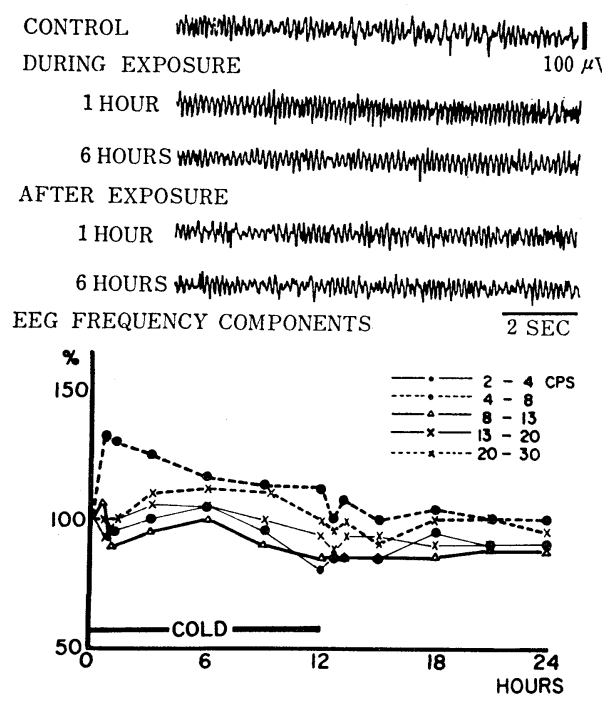

Fig. 3. Typical effects of cold exposure $\left(-20^{\circ} \mathrm{C}\right)$ on EEG activity of the posterior hypothalamus. The upper part of the illustration shows the EEG pattern before, during and after cold exposure. The lower part shows the change of EEG frequency components.

Note that the EEG is hyperactivated and that the $4-8 \mathrm{cps}$ component is markedly increased after 0.5 to 1 hour of cold exposure.

amplitude sinusoidal waves. Then the component began to gradually decrease, and despite continued cold exposure, it almost returned to the control after 6 to 9 hours. But other frequency components of 2-4 cps, 8-13 cps, 13$20 \mathrm{cps}$ and $20-30 \mathrm{cps}$, showed almost no change or a slight tendency to decrease. The initial change suggests increased activity of this region (FIG. 3).

Anterior hypothalamus

The medial preoptic area has been regarded as a center of heat dissipation. EEGs recorded from the lateral part and the medial part of this region show different basic patterns (FIG. 4). The former has a fast wave of 20-30 
cps as the basic wave. The EEG here hardly showed any change during the early phase of cold exposure. After 3 hours of exposure the basic component began to increase and after 12 hours of exposure, the increment was

\section{MED. PREOPTIC AREA}

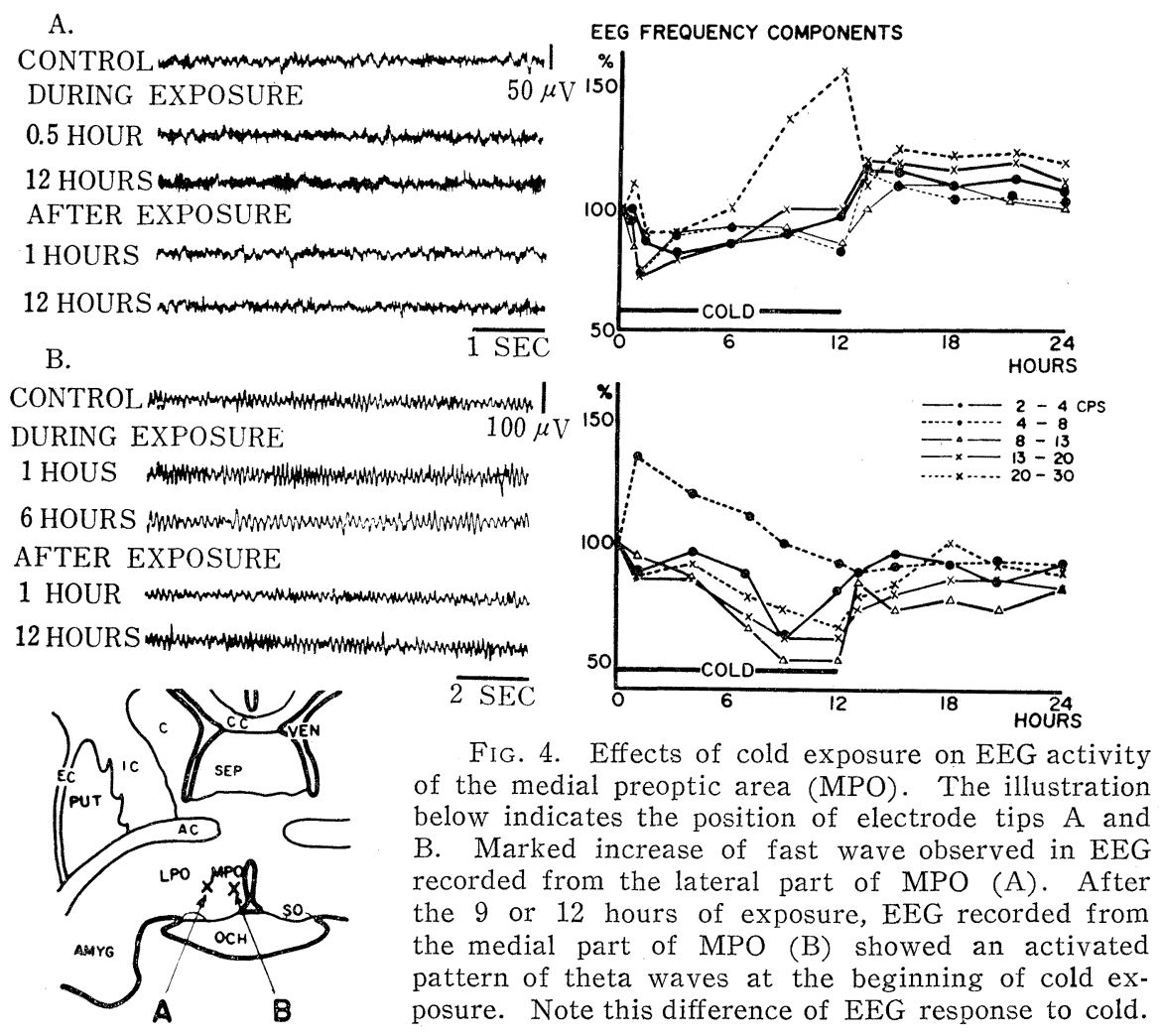

$48-60 \%$ of the control level. The EEG change was similar to that of the medial amygdala. Such an increasing tendency of the fast wave in the medial preoptic area was seen more remarkably in thyroidectomized rabbits. According to this phenomenon, the increasing tendency is supposed to be related to TSH secretion. Compared with other frequency components, it showed unappreciable change.

On the other hand, the basic wave of EEG recorded from the medial part of this region is a rhythmic theta wave. Synchronization and an increase in amplitude of the theta wave brought about by cold exposure. After half an hour or an hour, integrated band value of $4-8 \mathrm{cps}$ increased by 34 $46 \%$ of the control level. But thereafter, the frequency component showed a tendency to decrease and returned to the control level after 12 hours of exposure to extreme cold, 
The EEG of the periventricular stratum in the anterior hypothalamus surrounding the third ventricle shows a $4-8 \mathrm{cps}$ wave as the main component. This component increased gradually and slow waves less than $4 \mathrm{csp}$ decreased when the rabbit was exposed to cold. The increment of integrated band value of $4-8$ cps was $43-56 \%$ of the control level by $6-9$ hours of exposure. Other frequency components hardly changed during cold exposure.

Seasonal variation was hardly seen with respect to the alteration in EEG activity of the anterior hypothalamus.

\section{Basal hypothalamus}

SAWYER (1965) and KAWAKAMI (1966) reported that the ventromedial hypothalamus had a close relation to ACTH secretion. In every season EEG activity in this area was enhanced when a rabbit was exposed to extreme cold, and especially the $4-8 \mathrm{cps}$ of the theta wave components increased remarkably among all the frequency band components. After 6 hours of cold exposure, it reached the maximum (24-45\% increase), and kept this same level for 12 hours of cold exposure. When it was removed from the cold environment, the activity of the ventromedial nucleus returned to the control level within an hour. The time course of such a change in activity of the medial part of the basal hypothalamus was in accordance with the time course of biosynthesis of steroids in the adrenal cortex when a rabbit is exposed to extreme cold ${ }^{11}$. Therefore, this part is supposed to urge the releasing of $\mathrm{ACTH}$, in response to cold stress. It was hard to see distinct seasonal variation in the EEG activity of this part under cold exposure.

EEG recorded from the arcuate nucleus and its adjacent region which is a final common pathway for the central nervous control of the adenohypophysis was not affected by exposure to cold.

\section{Lateral hypothalamus}

EEG of the lateral hypothalamus showed a theta wave of $4-8 \mathrm{cps}$ as a basic wave in arousal state. The activity was markedly depressed during cold exposure. That is, all frequency components including the basic wave gradually decreased from the beginning of the exposure and the decrement of the basic component was $25-40 \%$ of the control level after 3 hours of exposure. Thereafter the reduction of EEG activity continued as long as the rabbit was exposed to cold.

Seasonal variation was not obvious in the activity of this region.

3. EEG activities of immature rabbit under cold exposure.

EEGs were recorded from the immature rabbit's brain whose mechanisms of body temperature control were still not well developed. Experiments were performed with rabbits of 14 to 40 days of age, and the results were compared with those of mature ones.

When the immature rabbits, younger than 15 days of age, were exposed 
to cold $\left(20^{\circ} \mathrm{C}\right.$ below zero), they showed more remarkable shivering than mature ones. Rectal temperature, however, fell immediately and reached $33 \pm 1.5^{\circ} \mathrm{C}$ by 4 hours of cold exposure. In accordance with the temperature decline, EEGs recorded from the hypothalamus and the limbic area flattened. But the EEG from the amygdala increased in amplitude even after the temperature declined below $34^{\circ} \mathrm{C}$. All frequency components were augmented. When the rectal temperature was below $32^{\circ} \mathrm{C}$, the amplitude of each frequency component tended to decrease with the decline in temperature and to flatten. Immediately after release from exposure, the activated pattern of the amygdala appeared again and it continued for more than 6 hours (FIG. 5). High

\section{AMYGDALA \\ IMMATURE RABBIT}
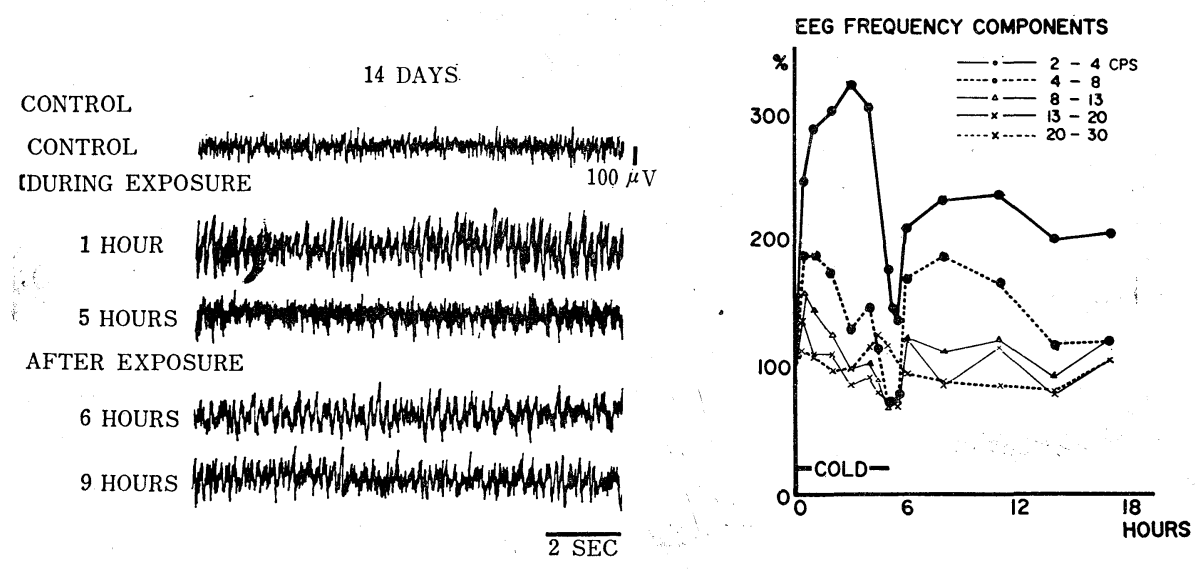

Fig. 5. Typical effects of cold exposure on amygdalar EEG activity of an immature rabbit (14 days of age).

Note that the amygdalar electrical activity is hyperactivated by cold exposure even when the rabbit is so young that the electrical activity of many other regions of the hypothalamus and limbic area is depressed under cold.

amplitude burst is often observed in EEGs recorded from the amygdala in respiratory rate, but in a cold environment the respiratory rate is greatly decreased for the prevention of heat loss. Therefore this amygdalar change due to exposure to cold is thought to be of some other origin.

Rectal temperature of rabbits about 20 days old showed a very gradual decline when the animals were exposed to cold (approximately $34^{\circ} \mathrm{C}$ after 6 hours of exposure), but it fell rapidly if the rabbits were exposed to cold for longer than 6 hours. The EEG in the hippocampus showed suppression of $4-8 \mathrm{cps}$ of sinusoidal wave along with the temperature decline. This change of the basic wave was transiently inversed; that is, the EEG was hyperac- 


\section{HIPPOCAMPUS}

\section{IMMATURE RABBIT}
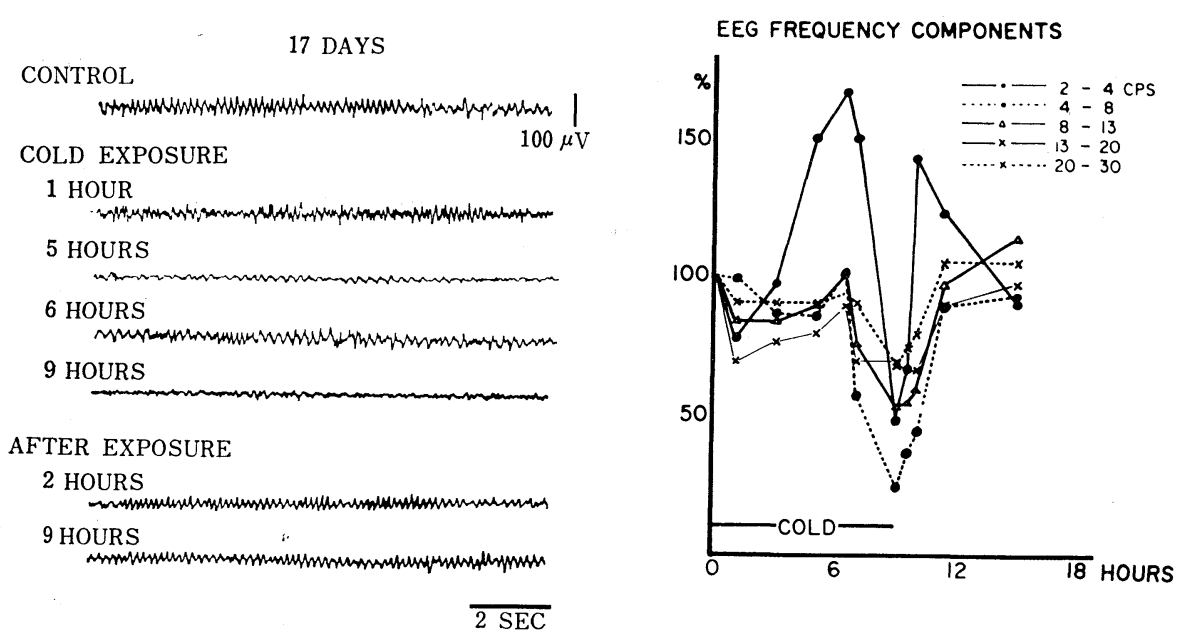

FIG. 6. A typical example of the change in EEG recorded from the hippocampus of an immature rabbit (17 days old).

Note that the hippocampal activity is gradually depressed in progress of exposure time, but just before the abrupt fall of body temperature (after 6 hours exposure), transient activation is observed.

tivated when the temperature declined to $32^{\circ} \mathrm{C}$. After that, the EEG pattern began to decrease in amplitude, till it replaced by flattening. EEG activity in the amygdala hardly showed such an enhancement under extreme cold as was characteristically observed in the rabbit younger than 18 days old.

When rabbits older than 30 days of age were exposed to extreme cold, any changes that occurred in the rectal temperature or the EEG activities of the hypothalamus and the limbic area, including the hippocampus and amygdala, were of the same nature as those in adult rabbits.

4. Effect of repetitive cold exposure on EEG activity.

Rabbits were exposed to the extreme cold of $20^{\circ} \mathrm{C}$ below zero for 12 hours per day and the effect of this repetitive exposure was investigated. This experiment was performed in mid-summer. Rectal temperature dropped by 0.4 to $1.0^{\circ} \mathrm{C}$ at the first exposure. However, no remarkable change was observed at the 3 rd exposure. The rectal temperature rose slightly ( 0.3 to $\left.0.5^{\circ} \mathrm{C}\right)$ at the 4 th or 5 th exposure.

The changes of the EEG activity of the hippocampus and the central grey at the 1st exposure were characterized by a remarkable increase of theta waves as was previously stated. These characteristics became less remarkable after repeated exposure. For example, the EEG activity in the hip- 
pocampus hardly changed under repeated exposures from the 5 th to the 7 th. As for the central grey, the increase of theta wave component was scarcely seen even at the 2nd exposure, and all frequency bands including the 4-8 cps component tended to decrease.

The EEG change of the medial amygdala at the 1st exposure was, as already described, an increase of the faster component between 20 and 30 cps accompanying a decreasing tendency of other frequency components. However, the increase of the fast wave component was less obvious at the 2nd exposure and no distinguishable changes were perceived in each frequency component up to the 7th exposure.

The EEG of the lateral amygdala presented a decrease of all frequency components at the first exposure, but this tendency gradually disappeared with the repetition of cold exposure and showed no remarkable change at the 6 th or 7 th exposure.

The characteristics of the EEG activity recorded from the medial preoptic area when animals were exposed to cold were the increase of the faster components of $20-30 \mathrm{cps}$. This was hardly perceived at the $3 \mathrm{rd}$ and 4 th exposure, while a decreasing tendency of the other frequency components still existed until the 7 th exposure. The increase of theta wave which was perceived on the EEG recorded from the periventricular stratum surrounding the 3rd ventricle in the anterior hypothalamus still appeared at the 7 th exposure but disappeared at the 10th exposure.

The EEG activity of the ventromedial hypothalamus was markedly increased by the first exposure, but the response to the cold stimulus was reduced at the 2nd or 3rd exposure. Finally, no effect was perceived at the 5th exposure. The EEG from the arcuate nucleus showed almost no change under the 1st exposure and remained the same even after repeated exposure.

The increase of the $4-8 \mathrm{cps}$ component and a slight decrease of the other frequency components were the characteristics of the EEG activity of the posterior hypothalamus under the 1st exposure. The former noticeably decreased even under the 2nd exposure, and the latter disappeared at the 6th and the 7 th exposure.

Both activities of the limbic areas and the hypothalamic areas eventually showed no reaction to cold exposure, but the former tended to lose reaction to cold exposure earlier than the latter.

5. Effect of electrical stimulation of the hypothalamus and the limbic areas on body temperature.

A stimulation experiment was carried out in order to elucidate the influence of increased activity in various parts of the brain on body temperature. Stimulation was given by square pulses whose frequencies were $100 \mathrm{cps}$ 

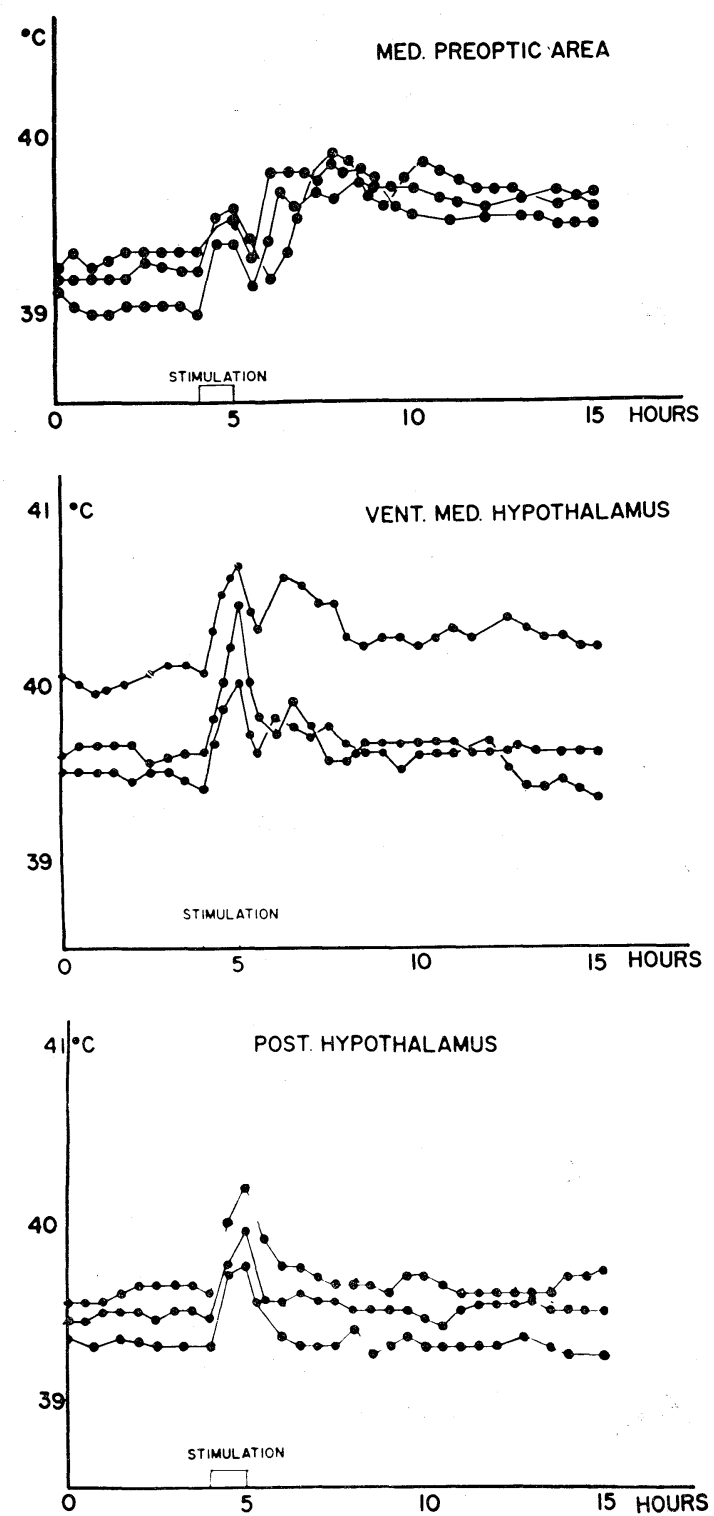

FIG. 7. Effects of electrical stimulation of the medial preoptic area, ventromedial hypothalamus and posterior hypothalamus (the so-called sympathetic zone of the hypothalamus) on rectal temperature. Stimulation; $100 \mathrm{cps}$ square wave pulse, $2.5-3.0 \mathrm{~V}, 0.5 \mathrm{msec}$. duration, for 1 hour, $60 \mathrm{sec}$. on and $60 \mathrm{sec}$. off.

Note that rectal temperature rises during stimulation of all of these areas (immediate effect) and rises again after stimulation of the former two (delayed effect). 

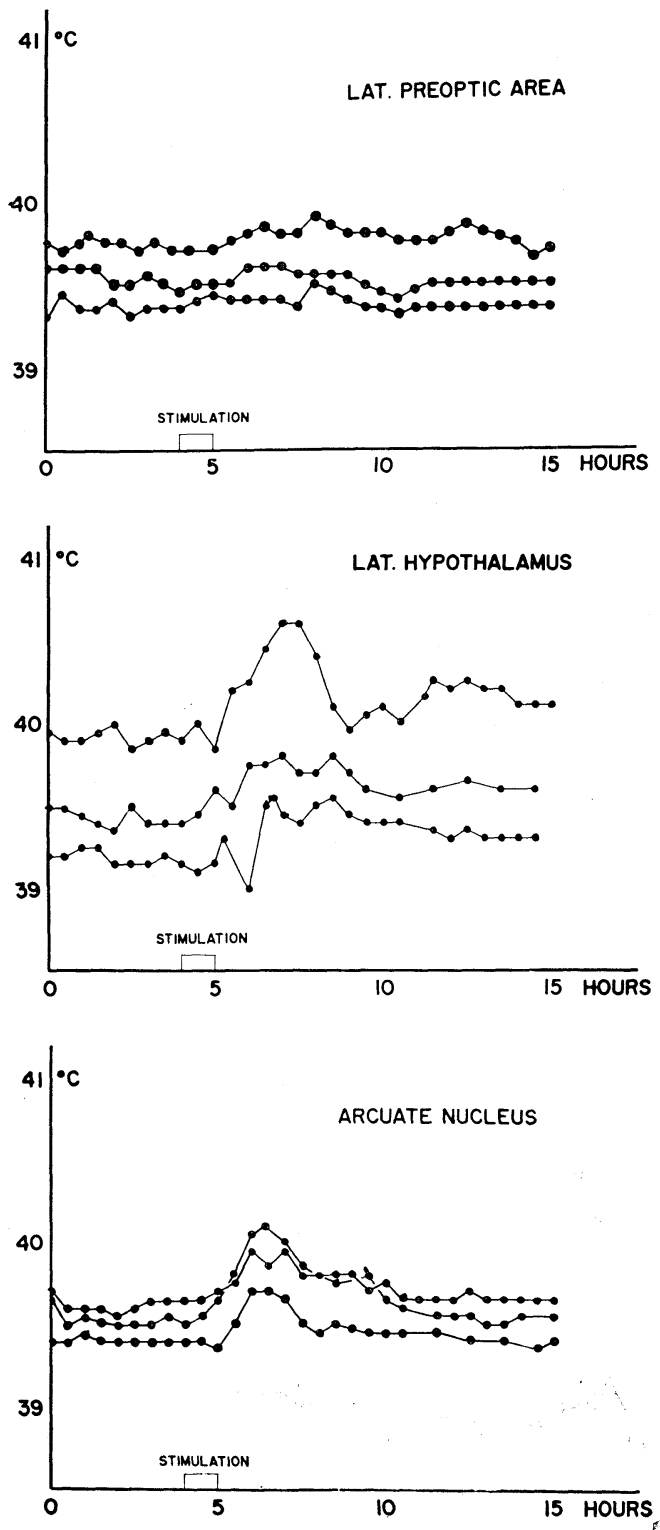

FIG. 8. Effects of electrical stimulation of the lateral preoptic area, lateral hypothalamus and arcuate nucleus (so-called parasympathetic zone) on rectal temperature. Parameters of stimulation is the same as FIG. 7.

Note that elevation of rectal temperature was absent during stimulation of these regions and was observed after stimulation of the latter two regions. 
with a duration of $0.5 \mathrm{msec}$. This was applied repeatedly for an hour by the method of one minute's stimulation and one minute's pause. The maximum intensity below EEG seizure threshold, determined by the preliminary experiment, was used for stimulation. Three to 5 rabbits were employed for each location of stimulation. The EEG was recorded simultaneously during stimulation and was examined to see if the activity of the stimulated part was really enhanced and if a seizure pattern appeared.

The medial preoptic area, ventromedial hypothalamus and posterior hypothalamus (which are all included in the so-called sympathetic zone of the hypothalamus) were stimulated with an intensity of 2.0-3.0 volts. During the stimulation of these areas, the rectal temperature rose by 0.3 to $0.8^{\circ} \mathrm{C}$, and after a period of about one hour tended to return to the prestimulation level. Moreover, among these regions, after stimulation of the ventromedial hypothalamus and the medial preoptic area, the rectal temperature rose again by $0.2-0.8^{\circ} \mathrm{C}$, but with a latent period of 20 minutes to 3 hours - so that it showed a biphasic change (FIG. 7). On the other hand, after stimulation of the posterior hypothalamus there was no delayed elevation in rectal temperature; so in this case, the change in temperature was monophasic (FIG. 7).

When the lateral hypothalamus, lateral preoptic area, periventricular stratum and arcuate nucleus (which are all in the so-called parasympathetic zone of the hypothalamus) were stimulated, hardly any rise in rectal temperature was observed during stimulation. However, after stimulation of the arcuate nucleus and lateral hypothalamus rectal temperature rose by $0.3-0.7^{\circ} \mathrm{C}$ (FIG. 8).

Electrical stimulation was also applied to the limbic areas. Soon after the stimulation of the medial amygdala by 2.0 to 2.5 volts, the body temperature rose and reached its maximum in half an hour to 2 hours after stopping the stimulation. The temperature rose by 0.3 to $0.4^{\circ} \mathrm{C}$ compared with the control level, and returned to the control level in 4 to 8 hours. When the lateral amygdala was stimulated, the body temperature rose and attained the maximum $\left(0.2-0.3^{\circ} \mathrm{C}\right)$ in $1.5-4.0$ hours after the stimulation was stopped (FIG. 9).

When the hippocampus was stimulated with an intensity between 0.5 and 0.8 volts, the body temperature fell by 0.15 to $0.25^{\circ} \mathrm{C}$ during the stimulation. After the stimulation, it gradually rose and reached the maximum 1.5 to 5.0 hours later. This was higher by 0.2 to $0.3^{\circ} \mathrm{C}$ than that of the control. It returned to the normal level in 5.5 to 9 hours later (FIG. 9). In an attempt to determine the pathway through which the activated impulses of the hippocampus stimulate the hypothalamus and consequently induce the elevation of the body temperature, the stria terminalis was lesioned. After the stria terminalis was bilaterally destroyed, the hippocampal stimulation brought about almost the same change of rectal temperature as that of the rabbit without 

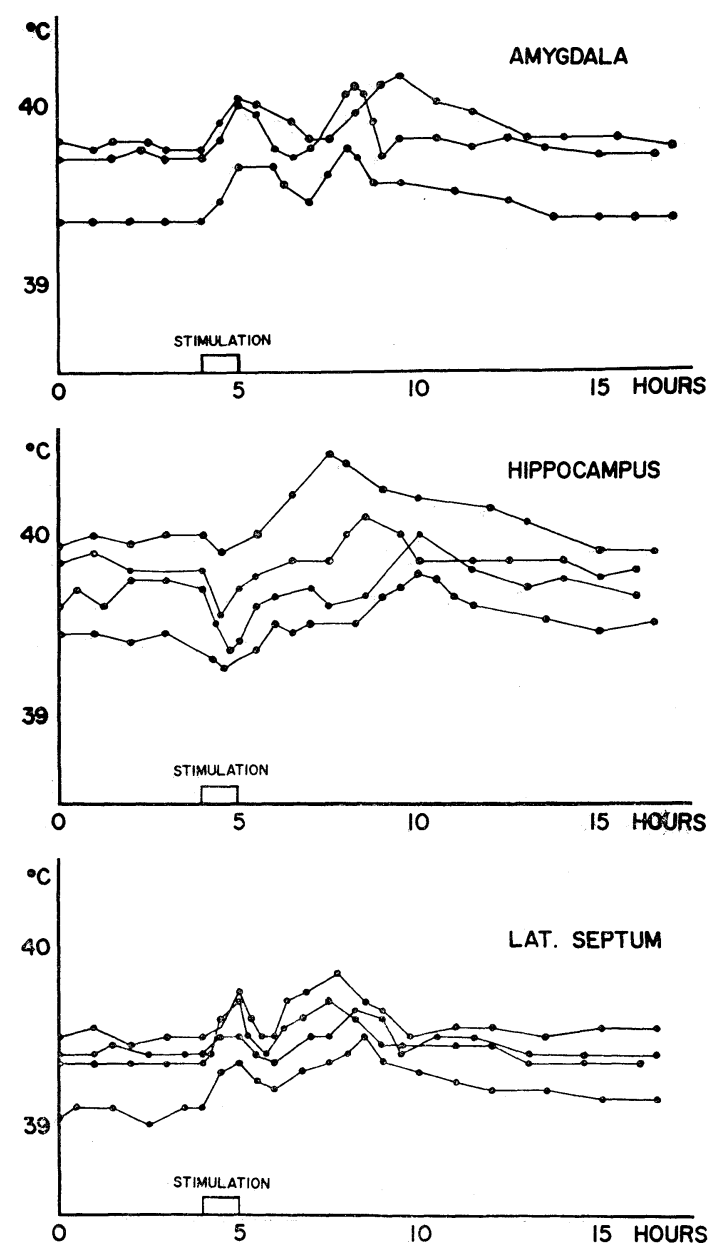

Fig. 9. Effects of electrical stimulation of the amygdala, hippocampus and lateral septum (limbic area) on rectal temperature.

Note the biphasic change of rectal temperature.

destruction of the stria terminalis. This fact suggests that the effect of hippocampal stimulation on body temperature is not due to the secondary excitation of the amygdala.

When the lateral septum was stimulated with an intensity of $2.0-2.5$ volts, the rectal temperature rose by 0.4 to $0.5^{\circ} \mathrm{C}$ during stimulation. After stimulation, it returned almost to the control level, and then rose again in 0.5 to 1.0 hour and reached the maximum $\left(0.25-0.35^{\circ} \mathrm{C}\right.$ higher than the control) in 2 to 5 hours. It returned to the control level in 3 to 7 hours (FIG. 9). When the medial septem was stimulated, the rectal temperature showed almost no change other than a fluctuation in the normal range $\left( \pm 0.1^{\circ} \mathrm{C}\right)$. 
The stimulation of each part of the brain caused an immediate and or a delayed effect in the rectal temperature. It may be supposed that the former was caused by neural factors or by fast-acting substances like epinephrine, and that the latter was brought about by slow-acting factors such as hormones released into blood stream. The latter factor may be significant because it might play an important role when the rabbits are exposed for a long time to extreme cold. In order to clarify the significance of this point, the following experiment was attempted.

5. Changes in body temperature caused by stimulation of the brain of the adrenalectomized and thyroidectomized animals.

Adrenocortical hormone and thyroid hormone, as well as epinephrine, are known to have a close relation to the regulation of body temperature. When these hormones (cortisone acetate $3 \mathrm{mg} 1 . \mathrm{m}$. , 1-thyroxine $\mathrm{Na} 20 \mathrm{mg}$ l.v.) were given to rabbits, it was found that the rectal temperature rose by $0.3-0.9^{\circ} \mathrm{C}$.

When the adrenalectomized rabbits were exposed to cold at the temperature of $-20^{\circ} \mathrm{C}$ for 6 to 8 hours their rectal temperature fell as low as $34^{\circ} \mathrm{C}$. However, the thyroidectomized rabbits did not manifest any considerable fall in rectal temperature (even in more than 3 weeks after thyroidectomy), although they were exposed to extreme cold for 12 hours.

\section{AFTER ADRENALECTOMY}
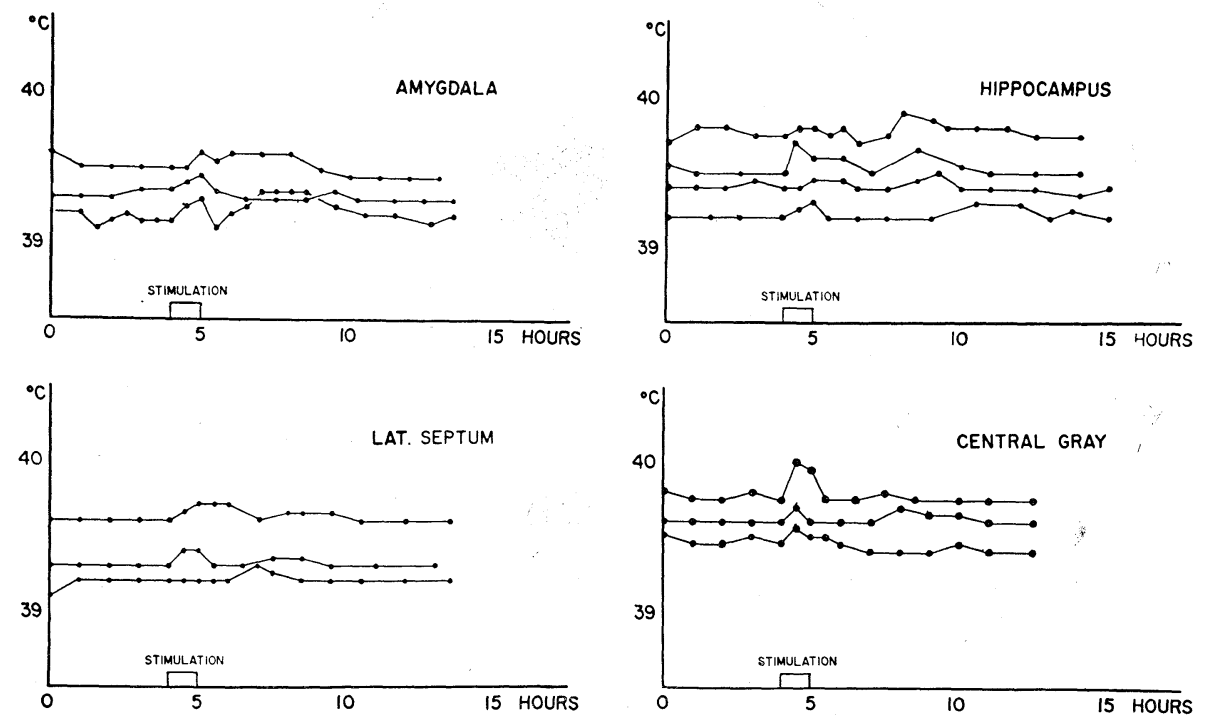

FIG. 10. Effects of electrical stimulations of some of the limbic area on rectal temperature in the adrenalectomized rabbits.

Note that the biphasic changes observed in the intact rabbits are noticeably decreased. 
The effects of stimulation on rectal temperature were absent or remarkably reduced in adrenalectomized rabbit (FIG. 10). On the other hand, when thyroidectomized rabbits were stimulated the delayed effects had almost the same intensity as intact animals.

6. Changes in body temperature caused by stimulation of the brains of rabbits with the arcuate nucleus lesion.

The arcuate nucleus and median eminence of the hypothalamus are known to be a common pathway of impulses which come from the limbic area and the other hypothalamic areas for the control of pituitary secretion. Rabbits in which the arcuate nucleus and adjacent region had been previously destroyed were observed with respect to the change in rectal temperature caused by stimulating the limbic area.

It was observed that stimulation of the limbic area caused no noticeable rise in rectal temperature. This fact suggests that the limbic-hypothalamopituitary system has an important role in effecting the rise of rectal temperature by stimulation of the limbic area.

\section{DISCUSSION}

In his cytoarchitectural study, GRÜNTHAL ${ }^{12)}$ divided the hypothalamus longitudinally from the 3 rd ventrocle outward, into three zones. KUROTSU ${ }^{13}$ made a further investigation and found that each of these divisions has a different type of autonomous function, and they called them a-parasympathetic zone, $b$-sympathetic zone and c-parasympathetic zone. The results of our experiment are discussed in the light of their theory as follows.

It was proved that during exposure to extreme cold the medial preoptic area, ventromedial hypothalamus and posterior hypothalamus, which belong to the b-sympathetic zone, showed increased activity. Moreover, the rectal temperature rose remarkably in response to stimulation of these areas. Therefore, it is reasonable to assume that the activity of the sympathetic zone in the hypothalamus plays an important role in the maintenance of body temperature in extreme cold. Even in the parasympathetic zone of the hypothalamus, however, some areas such as the lateral hypothalamus and suprachiasmatic nucleus showed increased activity in response to exposure to extreme cold, and stimulation of these areas resulted in a rise in body temperature. So it may be assumed that no only the sympathetic zone of the hypothalamus, but also some areas of the parasympathetic zone participate in the regulation of the body temperature.

According to the results of the stimulation experiments upon the several parts of the brain in this experiment, two types of rise in body temperature were found in response to a one hour period of on/off stimulation ( $30 \mathrm{sec}$. on $30 \mathrm{sec}$. off). One was an immediate effect and the other a delayed effect. 
The former may be attributed to neural factors or to fast-acting humoral factors such as epinephrine, and the latter may be attributed to slow-acting humoral factors such as adrenal hormones and thyroid hormones. The experiments using adrenalectomized or thyroidectomized rabbits proved that the pituitary-adrenal axis has an important role in thermo-regulation during exposure to extreme cold.

Thyroid hormone, epinephrine and adrenocortical hormones have been known to participate in the thermo-regulatory mechanism. KAWAKAMI et al. ${ }^{14)}$ and SHIzUmE ${ }^{15)}$ reported that the concentration of thyroid hormone in the blood was increased by electrical stimulation applied to limbic structures such as the hippocampus and amygdala.

The present results, indicating the activation of these regions during cold exposure, seem to represent the facilitation of thyroid function by the central nervous system.

$\mathrm{SATAKE}^{16)}$ and other researchers have confirmed the elevation of epinephrine concentration during cold exposure. This hormone has an action of mobilization of glucose from glycogen serving for heat production and at the same time causes capillary contraction preventing heat loss. GELLHORN ${ }^{17)}$ demonstrated that stimulation of the posterior hypothalamus induces sympatheticotonic symptoms. Epinephrine is also known to be released by stimulation of the posterior hypothalamus ${ }^{18}$. On the basis of previous studies such as PORTER ${ }^{19)}$, in which it is reported that the activity of the posterior hypothalamus is influenced by epinephrine, it is suspected that the hormone released into the blood by cold stimuli is feedback to this portion. Therefore it is inferred that excitation of the posterior hypothalamus would induce elevation of the concentration of epinephrine in the blood and at the same time the increased epinephrine in the blood feeds back to the posterior hypothalamus; that is, the posterior hypothalamus controls the epinephrine level in the blood. The posterior hypothalamus in also known to be related to production of shivering ${ }^{8)}$. The increased activity of the posterior hypothalamus under extreme cold may thus contribute to the maintenance of body temperature.

As to the role of the brain in the secretion mechanism of ACTH which is easily released on cold stimulus, KAWAKAMI et al. ${ }^{20)}$ reported that electrical stimulation of the amygdala and the hippocampus effectively enhanced adrenocortical activity. Since secretion of the adrenocortical hormones is facilitated by stimulation of the basal part of the medial hypothalamus, and inhibited by making lesions or implanting minute amounts of crystalline hydrocortisone in the same portion, this portion of the hypothalamus has been considered as a center controlling the release of adrenocortical hormones in a negative feedback manner. The present knowledge that the EEG activity of the medial amygdala, hippocampus and ventromedial hypothalamus 
is elevated during cold exposure may support and add to the view which considers the basal hypothalamus as a focus for the negative feedback control of the ACTH releasing mechanism. From these results we may draw the following conclusion. In the early stage of exposure, the fast-acting factors, such as sympathetic nerves, adrenalin, etc., work for the maintenance of body temperature. Later when exposure is prolonged, the limbic-hypothalamo-pituitary-adrenal axis and the limbic-hypothalamo-pituitary-thyroid axis cooperate for the maintenance of body temperature. The former, especially, plays a most important part in this maintenance.

It is confirmed that there exists an anatomical fiber connection from the hippocampus or lateral septum to the central grey and posterior hypothalamus. The present experiment revealed that alteration and its time course of EEG activity in these regions during cold exposure resembled each other. That is, in these region synchronization of the theta rhythm characterized the EEG pattern during the first 6-9 hours of cold exposure, which then gradually returned to the control level in the next 3 hours. Thus, electrical activity of the hippocampus, central grey, lateral septum and posterior hypothalamus is elevated during the early phase of cold exposure. On the other hand, the amygdala and the medial preoptic area showed similar alteration of activity during cold exposure. Although these regions seemed to be activated by cold exposure, the change appeared rather gradually and attained its maximum 9-12 hours after the start of exposure. The relatively phasic response of the hippocampus, central grey and posterior hypothalamus to cold exposure and the tonic response of the amygdala and the medial preoptic area may be assumed to reflect the functional differentiation in the defense mechanism against extreme cold.

It is established that the amygdalar stimulation elevates the body temperature $^{21,10)}$ and induces a group of responses which facilitate heat production and prevent heat loss by such mechanisms as elevation of blood sugar, piloerection of inhibition of respiration. As described before, in an immature rabbit with undeveloped regulation of body temperature, the amygdala was the earliest structure to show activation by cold exposure, and the younger the subject examined the more remarkable the amygdalar activation in response to cold exposure. Therefore it may be assumed that the amygdala is ontogenetically the most primitive center for the regulation of body temperature. Activation of the amygdala caused by exposure to cold was observed at younger ages than hippocampal hyperactivity. And when the hippocampal activation appeared under extreme cold, the amygdalar was reduced. It may be assumed from this fact with the progress of development, the hippocampus comes to have an important role in thermo-regulation in place of the amygdala. In an immature rabbit, a phasic but remarkable activation of the hippocampus preceded an abrupt decline of body temperature. As already 
discussed, adult hippocampus also shows a phasic activation at the early phase of cold exposure, but without decline of body temperature. Considering out previous results that excitation of the hippocampus facilitates adrenocortical and thyroid activity, the phasic activation of this region may have significance as an emergent reaction for the maintenance of body temperature. This inference seems to be supported by our result that hibernating animals, like a ground squirrel (tamias cibiricus), wake up from hibernation, elevation of the body temperature is preceded by phasic hyperactivation of the hippocampus $^{22)}$.

The experiment on the change in body temperature under cold exposure showed that in summer the body temperature was lowered while in winter or after repeated exposure to cold it was, on the contrary, elevated. This fact may be interpreted as showing that in winter or after repeated exposure to cold, the animal was acclimated enough so that the temperature regulation is not disorganized by extreme cold. It was also found that the EEG activity of the limbic system is more remarkable affected in summer or at the first exposure to cold stimulus than in winter or after repeated exposure. Analogous phenomena have been reported on peripheral organs. For example, in a cold acclimated mouse depletion liver glycogen is markedly less than in an original case ${ }^{23)}$.

Thus it is assumed that after acclimation to cold environment, activation of the limbic structures is unnecessary and temperature control can be carried out without it.

\section{SUMMARY}

From the viewpoint of the neurohumoral interaction in the control of body temperature, brain activity under extreme cold was studied by means of EEG observation and electrical stimulation, employing New Zealand white rabbits with chronically implanted bipolar electrodes in the several parts of the brain. During experiment, rabbits were free to move around on the observation table or in the cold box under constant illumination.

The results were as follows:

The EEG activity showed the following alterations during exposure to cold (at a temperature of $20^{\circ} \mathrm{C}$ below zero for a period of 12 hours).

1. Exposure to the extreme cold brought about an enhancement of EEG activity in the medial preoptic area, ventromedial nucleus of the hypothalamus, posterior hypothalamus, periventricular stratum, hippocampus, medial amygdala, lateral septum and central grey, and suppression of EEG activity in the lateral preoptic area, lateral hypothalamus, lateral amygdala and medial septum. EEG recorded from the arcuate nucleus and its adjacent region did not show any notable change resulting from cold exposure. To 
summarize the above results, EEG of the areas belonging to the so-called sympathetic zone showed elevated activity under extreme cold. At the same time, some areas belonging to the so-called parasympathetic zone and the limbic system also showed an activated pattern during cold exposure. EEGs were recorded from the brain of immature rabbits of 14 to 40 days of age. It was suggested that the amygdala was the earliest center to develop for the regulation of body temperature. In the later stages of development the hippocampus takes the place of the amygdala in the thermo-regulatory mechanism.

2. Repetitive cold exposure $\left(20^{\circ} \mathrm{C}\right.$ below zero for 12 hours per a day) and simultaneous EEG recording were carried out for a period of 7 or 10 days in summer. The change observed in the hippocampus, amygdala, medial preoptic area, periventricular stratum, ventromedial hypothalamus and posterior hypothalamus observed at the initial exposure gradually decreased, until the EEG activity in these areas was hardly influenced by the 5th or 10th exposure to cold. The activity of the limbic areas tended to lose reaction to cold exposure earlier than the latter.

3. In order to elucidate the relation of increased activity in various parts of the brain to body temperature, a stimulation experiment was performed. Stimulation consisted of monophasic square wave pulses delivered unilaterally for one hour, $60 \mathrm{sec}$. on and $60 \mathrm{sec}$. off, at $0.5 \mathrm{msec}$. duration, $60 \mathrm{cps}$. The stimulation on each part of the brain caused a rise in rectal temperature. The change was divided into immediate effect (Effect during stimulation) and delayed effect (effect after stimulation). Immediate effect (rise of rectal temperature by 0.3 to $0.8^{\circ} \mathrm{C}$ ) was observed by the stimulation of regions included in the so-called sympathetic zone of the hypothalamus such as the medial preoptic area, ventromedial hypothalamus and posterior hypothalamus and some areas of limbic system such as the amygdala, lateral septum and central grey. On the other hand, delayed effect was observed by the stimulation of the medial preoptic area, ventromedial hypothalamus (so-called sympathetic zone), lateral hypothalamus, arcuate nucleus (so-called para sympathetic zone), hippocampus, amygdala, lateral septum and central grey (limbic area). It may be supposed that the immediate effect was caused by neural factors or by fast-acting substances like epinephrine, and that the latter was brought about by slow-acting factors such as adrenocortical hormones or thyroid hormone.

4. In order to clarify the significance of the delayed effect which might play an important role in the prolonged exposure to cold, electrical stimulation of the limbic area was carried out in adrenalectomized rabbits and thyroidectomized rabbits. The effect of stimulation on rectal temperature was absent or remarkably reduced in adrenalectomized rabbits, while it was observed in thyroidectomized rabbits in the same manner as in intact rabbits. 
5. Stimulation was applied to the limbic area of the rabbits whose arcuate nucleus and its adjacent region had been destroyed, since this area is a common path for impulses which come from the limbic area and other hypothalamic areas for the control of pituitary secretion. The rabbits showed no noticeable change of rectal temperature by the stimulation. From this fact it was suggested that the limbic-hypothalamo-pituitary sytem has an important role in the delayed rise of rectal temperature.

The authors wish to thank Mr. Robert L. CASADY, Mr. David Whitmoyer and Mrs. Virginia Hoover for their kind help in English expression.

This experiment was supported by a grant from the Ministry of Education, Japan.

\section{REFERENCES}

1) Barbour, H.G. (1912). Die Wirkung unmittelbar Erwärmung und Abkuhlung der Wärmezentra auf die Körpertemperatur. Arch. Exper. Path. u. Pharmakol., $70,1$.

2) Magoun, H.W., Harrison, F., Brobeck, J.R. and Ranson, S. W. (1938). Activation of heat loss mechanism by local heating of the brain. J. Neurophysiol., $1,101$.

3) Hardy. J.D., Fusco, M. and Hammel, H. T. (1958). Responses of conscious dog to local heating of anterior hypothalamus. The Physiologist, 1, 34.

4) Nakayama, T., Eisenman, J.S. And Hardy, J.D. (1961). Single unit activity of anterior hypothalamus during local heating. Science, 134, 560.

5) Murakami, N., Cunningham, D. J., Slolur JK, J. A. J. and Hardy, J.D. (1966). Interneurones in the temperature sensitive preoptic region. The Physiologist, 9, 250.

6) Anderson, B., Grant, R. and Larsson, S. (1956). Central control of heat loss mechanism in the goat. Acta physiol. scand., 41, 90-100.

7) Anderson, B. and Persson, N. (1957). Pronounced hypothermia elicited by prolonged stimulation of the heat loss centre in un-anesthetized goats. Acta physiol. scand., 41, 277-283.

8) Hemingway, A., Forgrave, P. And Birzis, L. (1954). Shivering supression by hypothalamic stimulation. J. Neurophysiol., 17, 375-386.

9) Katayama, J. (1965). Evaluation of amygdaloid nuclei in body temperature regulation. Rep. I. Effect of electrical stimulation of amygdaloid nuclei on rectal temperature in rabbit. Bull. the institute of constitutional med., 16, 51-63, (in Japanese).

10) Kolkegami, H., Kushiro, H. And Kimoto, A. (1955). Studies on the amygdaloid nuclei and periamygdaloid cortex. Experiments on gastro intestinal movement and body temperature in cat. Fol. psychiatr. neurol. jap., 8, 336-370.

11) Kawakami, M. And Seto, K. unpublished data.

12) Grünthal, E. (1929). Der Zellenplan des Hypothalamus beim Hunde. Zeits. Neur., 120, 157-177.

13) Kurotsu, T. (1957). Hypothalamus. In Clinical and pathological physiology. Nakayama-shoten Co., 8, 255 (in Japanese).

14) Kawakami, M. AND Negoro, H. (1967). Interaction between thyroid function and the activity of the limbic system. Proc. XXXX annual meeting of Japan endocrinological society, 152. 
15) Shizume, K., Matsuda, K., Kurie, M., Jino, M., Ishil, S., Nagataki, S., Matsuzaki, F. AND OKinaKa, S. (1962). Effect of electrical stimulation of the hypothalamus on thyroid function. Endocrinology, 70, 298-302.

16) Satake, Y. (1955). Secretion of adrenalin and sympathins Nanzando Co., Ltd.

17) Gellhorn, E. (1957). Autonomic imbalance and the hypothalamum. Univ. of Minnesota Press.

18) Goldfien, A. And Ganong, W.F. (1962). Adrenal medullary and adrenal cortical response to stimulation of diencephalon. Am. J. Physiol., 202, 205-211.

19) Porter, R.W. (1952). Alterations in electrical activity induced by stress stimuli. Am. J. Physiol., 169, 629-632.

20) Kawakami, M., Seto, K., Terasawa, E., Yoshida, K., Miyamoto, T., SekiGUCHI, M., AND HATTORI, Y. Influence of electrical stimulation and lesion in limbic structure upon biosynthesis of adrenocorticoid in the rabbit. Neuroendocrinology. In press.

21) KsADA, B. R. (1951). Somatomotor, autonomic and electrocorticographic responses to electrical stimulation of rhinencephalic and other structures in primates, cat and dog. Acta physiol. scand., 24, suppl. 83.

22) Kawakami, M., Yamaoka, S. And Negoro, H. (1966). Influence of cold exposure and brown adipose tissue upon EEG activity. J. biometeorological society of Japan, 1, 4. (in Japanese).

23) Smith, R. D. And Holker, D. J. (1962). Metabolism and cellular function in cold acclimation. Physiol. Rev., 42, 60-142. 